\title{
Review of mifamurtide in the treatment of patients with osteosarcoma
}

\author{
This article was published in the following Dove Press journal: \\ Therapeutics and Clinical Risk Management \\ II June 2010 \\ Number of times this article has been viewed
}

\author{
Leo Kager' \\ Ulrike Pötschger ${ }^{2}$ \\ Stefan Bielack ${ }^{3}$ \\ 'St. Anna Children's Hospital, Vienna, \\ Austria; ${ }^{2}$ Children's Cancer Research \\ Institute, Vienna, Austria; ${ }^{3}$ Klinikum \\ Stuttgart, Olgahospital, Pediatrics \\ 5 - Oncology, Hematology, \\ Immunology, Stuttgart, Germany
}

\begin{abstract}
Osteosarcoma is the most common primary malignant tumor of bone. The disease, however, is very rare with less than 2,000 expected patients at all age groups per year within the European Union and the United States of America. With multimodal therapy, which combines multiagent chemotherapy and complete resection of all macroscopically detectable tumors, about $60 \%-70 \%$ of patients with localized osteosarcoma can be cured. The prognosis, however, is still poor for patients with synchronous or metachronous metastatic or nonresectable primary disease, with reported 5-year event-free survival (EFS) rates of less than $30 \%$. Overall, the EFS rate has been rather stable since the introduction of combination chemotherapy including doxorubicin, cisplatin, high-dose methotrexate with leukovorin rescue, and/or ifosfamide. Mifamurtide, a modulator of innate immunity, which activates macrophages and monocytes, which in turn release chemicals with potential tumoricidal effects, may help to control microscopic metastatic disease and has been safely given together with standard adjuvant chemotherapy to patients with high-grade osteosarcoma. Results of the recently published intergroup study 0133 trial from the Children's Cancer and Pediatric Oncology Groups suggest that mifamurtide is a medicine that deserves further investigation in this orphan disease.
\end{abstract}

Keywords: orphan drug, orphan disease, outcome innate immunity, immunotherapy

\section{Introduction}

Osteosarcoma is the most common primary malignant tumor arising in bone. The incidence varies with age, and the Surveillance, Epidemiology and End Results (SEER) program of the National Cancer Institute provides the following osteosarcoma incidence data for the United States: 0-24 years, 4.4 cases per million per year; 25-59 years, 1.7 cases per million per year; and $\geq 60$ years, 4.2 cases per million per year. ${ }^{1}$ Similar data have been reported for Europe. ${ }^{2}$ There are two peaks in incidence: the first one during the pubertal skeletal growth spurt (15-19 years, 8.2 cases per million per year) and the second one during old age (75-79 years, 6 cases per million per year); and the latter is attributed to the sarcomatous transformation of Paget disease of bone and other benign bone lesions. ${ }^{1}$ A rare or orphan disease affects fewer than 5 people per 10,000; therefore, osteosarcoma has been named an ultraorphan disease. ${ }^{3}$ Given a population of about 385 million people in the European Union (EU) and 307 million people in the United States, less than 2,000 patients are expected to be diagnosed with osteosarcoma per year within the EU and the United States, and more than half of these patients are younger than 20 years.

Up to $20 \%$ of patients with osteosarcoma present with clinically detectable metastatic disease at diagnosis (synchronous metastases).$^{4}$ It is assumed, however, that about
Correspondence: Leo Kager

St. Anna Children's Hospital,

Kinderspitalgasse 6,A-1090 Vienna,

Austria

Tel $+43-1-40170-1250$

Fax +43-I-40I70-7000

Email leo.kager@stanna.at 
$90 \%$ of patients will have subclinical microscopic metastatic disease at the time of diagnosis of osteosarcoma.

The management of patients with osteosarcoma is complex and aims to completely remove all clinically detectable tumors surgically and control microscopic metastatic disease via systemic polychemotherapy. Complete surgical resection of all clinically detectable tumors is an essential prerequisite for long-term survival, and a complete remission can be achieved in up to $90 \%$ of patients with newly diagnosed osteosarcoma during frontline therapy. ${ }^{5}$ The use of intensive neoadjuvant and adjuvant chemotherapy has improved relapse-free survival rates in patients with localized extremity tumors from less than $20 \%$ with surgery only, to about $70 \%$ with a combination of surgery and multiagent chemotherapy. ${ }^{6}$ Despite aggressive multimodal treatment, patients having synchronous and metachronous metastatic disease with local relapse and nonresectable primary disease still have poor outcomes with survival rates of less than $30 \%$. $^{4,5,7,8}$

Surgical techniques have improved during the last decades, and there has been a successful shift from amputations toward limb-salvage surgery in neoadjuvant osteosarcoma trials. ${ }^{6}$ However, this progress in surgery did not significantly improve survival.

There are currently 4 chemotherapeutic agents with wellestablished efficacy in treating osteosarcoma: doxorubicin, cisplatin, high-dose methotrexate with leucovorin rescue, and ifosfamide. Since the introduction of standard chemotherapy regimens that include these drugs, the overall survival (OS) rates are stable, and no new drugs with proven efficacy have been added into the standard therapeutic armamentarium.

The immunomodulator mifamurtide (liposomal muramyl tripeptide phosphatidyl ethanolamine [L-MTP-PE] or MEPACT ${ }^{\circledR}$; Takeda Pharmaceutical Company Limited [Osaka, Japan] and Millennium: The Takeda Oncology Company [Cambridge, MA]), which activates macrophages and monocytes, has been safely given together with standard adjuvant chemotherapy in high-grade osteosarcoma patients aged between 2 and 30 years and has been reported to improve outcome in this ultraorphan disease.

Exhaustive, excellent reviews on mifamurtide and osteosarcoma are available for readers who seek detailed information., ${ }^{3-11}$ This article briefly reviews the use of mifamurtide in the treatment of patients with osteosarcoma, including a short discussion on the development of the concept of immunotherapy of cancer and the immunology of L-MTP-PE and a discussion on whether the current knowledge and data are robust enough to universally adopt the cost-intensive treatment with mifamurtide as standard of care for patients with osteosarcoma in the industrialized world.

\section{From Coley's toxins to mifamurtide}

More than 100 years ago, the bone sarcoma surgeon William Coley established the concept of immunotherapy of cancer by demonstrating that the infection produced by the injection of streptococcal organisms into a patient with cancer caused shrinkage of the malignancy. However, Coley's results were controversial, and the used endotoxins or bacterial products sometimes led to lethal side effects. With the advent of radiotherapy and chemotherapy, interest in Coley's mixed toxins waned. The concept that the immune system could be triggered by endotoxins or bacterial products to kill tumor cells had, however, been rediscovered, particularly in malignant diseases that do not respond sufficiently to conventional treatments.

It was found that not endotoxins or bacterial products themselves but factors released by the host's immune system (eg, macrophages) in response to the endotoxins or bacterial products can promote tumor necrosis, and one of the first identified factors was named tumor necrosis factor (TNF). Several other factors, collectively named cytokines and chemokines (secreted proteins with growth, differentiation and activation functions that regulate the nature of immune responses), were identified to promote toxic effects on tumor cells. Moreover, the receptors for these molecules and their intracellular signaling transduction pathways (such as the nuclear factor-kappaB [NF- $\kappa \mathrm{B}]$, the mitogenactivated protein kinases [MAPKs], the adaptor protein-1, and caspase 3 pathways) - which can promote cell survival, apoptosis, or inflammation reactions depending on the cellular context - have been identified. ${ }^{12}$

In parallel, research on innate immunity revealed that immunity against microbes relies on the specific host-receptor detection of pathogen- and danger-derived molecular signatures by macrophages and monocytes, with the subsequent activation of cellular signaling pathways such as NF- $\mathrm{KB}$ and MAPK pathways, which can promote inflammation and release of antimicrobial peptides. These pattern-recognition molecules encompass several families including the toll-like receptor (TLR) and the nucleotidebinding and oligomerization domain (NOD)-like receptors (NLRs such as NOD1, NOD2, and NLRP3). ${ }^{13}$ NOD1 and NOD2 comprise a subfamily of NLRs, which recognize distinctive core motifs within the peptidoglycan polymer in the cell wall of bacteria and possess an aminoterminal caspase recruitment domain, which is required to trigger NF- $\kappa \mathrm{B}$ signaling. ${ }^{13}$ 
The intracellular pattern-recognition molecule NOD2 detects the pattern of muramyl dipeptide (MDP), a motif present in all gram-positive and gram-negative bacterial walls. ${ }^{13}$ Mifamurtide (L-MTP-PE) is a fully synthetic lipophilic derivate of MDP, and therefore, it is most likely also a ligand and an activator of NOD2 and other targets of MDP. ${ }^{3}$ NOD2 has a tissue-specific expression pattern, and its expression is mainly restricted to monocytes, macrophages, dentritic cells, and intestinal Paneth cells. ${ }^{14}$ Recognition of MDP by NOD2 mainly results in the production of proinflammatory cytokines like interleukin-1 $\beta$ (IL-1 $\beta$ ), interleukin-6 (IL-6), and TNF- $\alpha$, and other molecules (eg, chemokines, antimicrobial peptides, adhesion molecules, etc) whose expression is under the control of NF- $\kappa B .{ }^{13}$ Moreover, MDP activates NLRP3, which is an essential component of the inflammasome, a protein complex that promotes the cleavage of procaspase 1 into its active form..$^{15}$ Active caspase 1 in turn is essential to activate proinflammatory cytokines like IL-1 $\beta$. Overall, activation of NLRs in macrophages and monocytes via MDP (or L-MTP-PE?) results in inflammation, release of antimicrobial peptides, fever, dendritic cell recruitment, polarization of T-helper cells, and promotion of bactericidal and potential tumoricidal effects. A scheme that depicts the possible mechanism of action of L-MTP-PE is provided in Figure 1.

\section{Pharmacology of mifamurtide}

The MDP molecule was found to be too pyrogenic and arthrogenic to be pharmacologically used in humans. Efforts focused on the development of less pyrogenic derivates, and in the early 1980s, L-MTP-PE was developed. The international nonproprietary name of the drug is mifamurtide. Mifamurtide, also called L-MTP-PE, is a liposomal formulation of the active ingredient MTP-PE, which is a fully synthetic, less pyrogenic, and longer-acting derivative of MDP. ${ }^{3}$ The liposomal encapsulation of MTP-PE has been shown to strongly enhance the tumoricidal effects of MTP-PE via macrophage activation in vitro. ${ }^{16}$ Moreover, there is evidence that liposomal encapsulated MTP-PE is about 10 times less toxic than MTP-PE. ${ }^{17}$ The molecular structure of L-MTP-PE is provided in Figure 2.

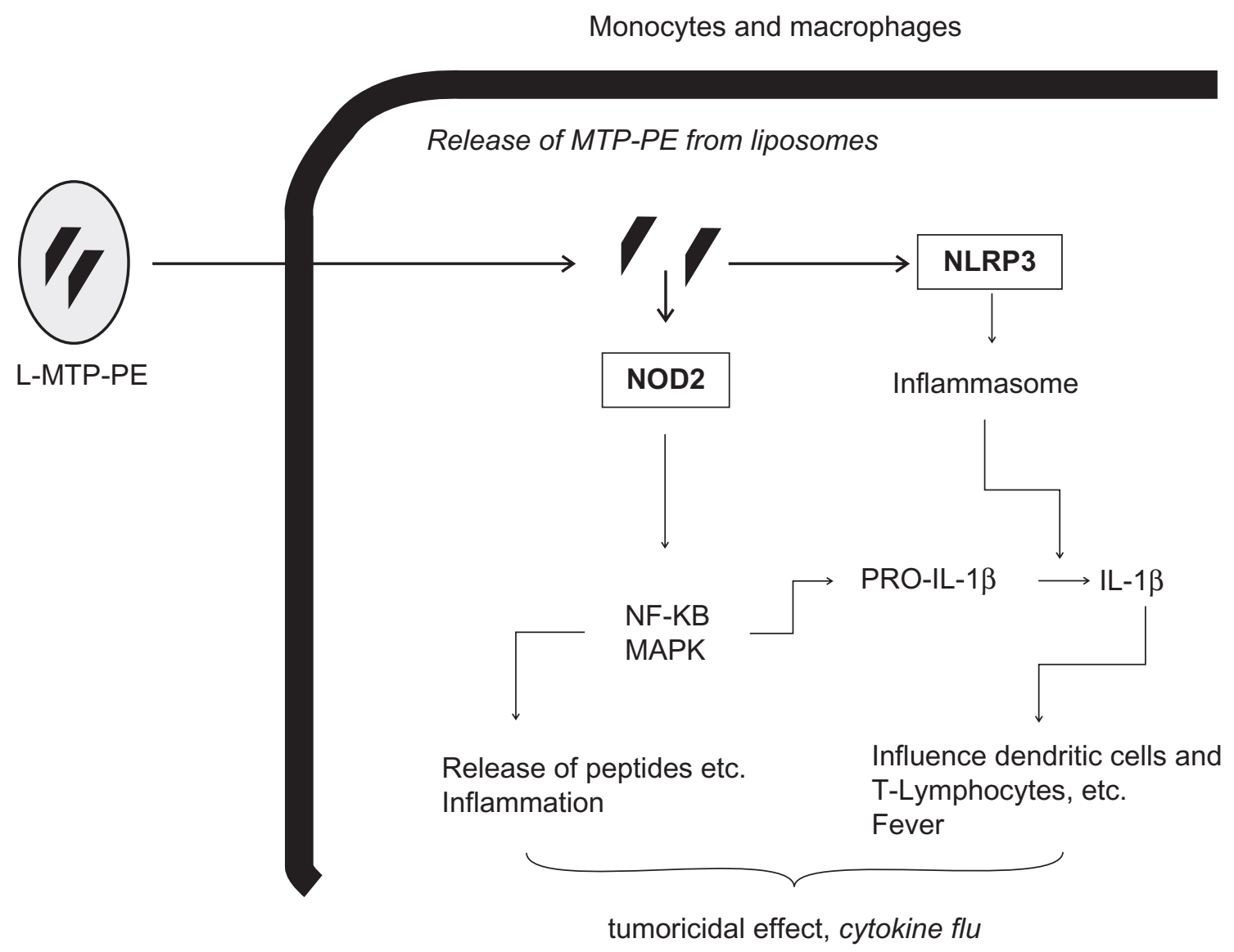

Figure I Possible mechanisms of action of L-MTP-PE (for details, see text).

Abbreviations: L-MTP-PE, liposomal muramyl tripeptide phosphatidyl ethanolamine; MAPK, mitogen-activated protein kinases; NF-KB, nuclear factor-kappaB; NLRP3, nucleotide-binding and oligomerization domain (NOD)-like receptor P3; NOD2, nucleotide-binding and oligomerization domain 2; IL-I $\beta$, interleukin I $\beta$. 


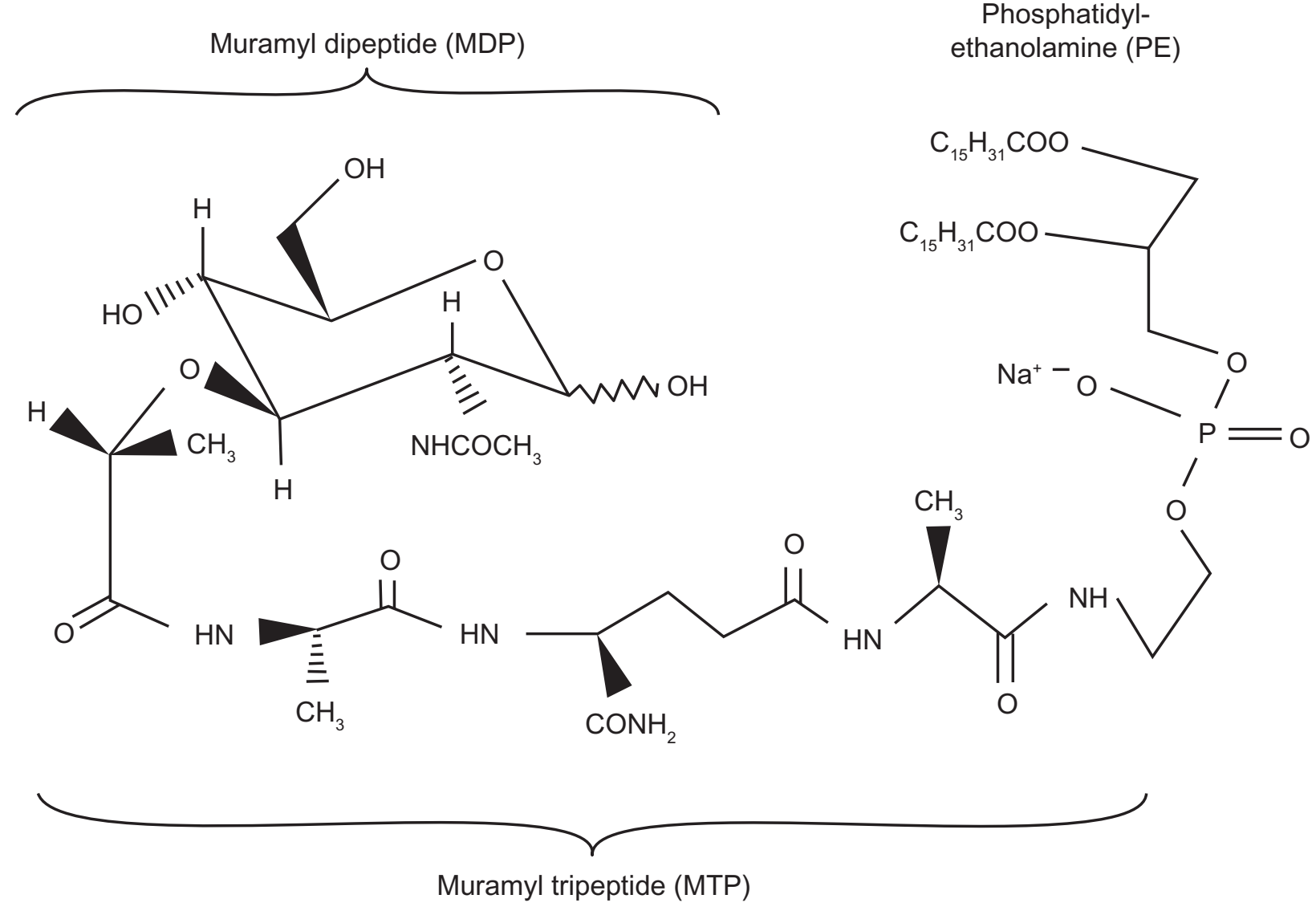

Figure 2 The molecular structure of liposomal muramyl tripeptide phosphatidyl ethanolamine (L-MTP-PE).

\section{Pharmacokinetics}

After intravenous (IV) infusion, mifamurtide is cleared very rapidly from plasma in cancer patients and in healthy adults, and the liposomes are mainly phagocytosed by the cells of the reticuloendothelial system (RES). ${ }^{3}$ In a biodistribution investigation in patients with osteosarcoma, ${ }^{99 \mathrm{~m}} \mathrm{Tc}$-labeled L-MTP-PE was found 6 hours after IV infusion in liver, spleen, nasopharynx, thyroid, and, to a lesser extent, in the lung, and this distribution pattern is consistent with that of the RES. ${ }^{18}$ Of note, in 2 of 4 patients with osteosarcoma lung metastases, radioactivity was detected at the site of the metastases. ${ }^{18}$ There is no evidence of accumulation of L-MTP-PE or free MTP-PE. ${ }^{19}$ More details on the pharmacokinetics of L-MTP-PE are available at the Web site of the European Medicines Agency (EMA) (http://www.ema. europa.eu/humandocs/PDFs/EPAR/mepact/H-802-PI-en. pdf; accessed 10.03.2010) and in an excellent recent review by Dr Paul Meyers. ${ }^{3}$

\section{Pharmacodynamics}

In animal studies, L-MTP-PE was found to be most effective against microscopic metastases but not against bulky disease. ${ }^{20,21}$ However, the exact mechanism by which L-MTP-PE induces a tumoricidal effect on osteosarcoma micrometastases is unknown. L-MTP-PE most likely activates macrophages and monocytes via the same signaling pathways as does MDP (ie, recognition via NOD2 and NLRP3 and activation of NF- $\mathrm{KB}, \mathrm{MAPKs}$, the inflammsome, etc), and the net effect can be the release of cytokines, proinflammatory molecules, etc, which have the potential to destroy tumor cells. In patients with osteosarcoma and healthy adults, it is well documented that within hours after L-MTP-PE was given, plasma levels of proinflammatory molecules, such as TNF- $\alpha$, IL- 6 , and IL- $1 \beta$, and other indicators of immune stimulation like C-reactive protein and neopterine were elevated. ${ }^{3,22,23}$ Clinically, a cytokine flu with fever, chills, and rigors can be observed due to the release of these proinflammatory molecules, especially after the initial administration(s) of L-MTP-PE, before the development of tolerance. $^{3}$

It is noteworthy that proinflammatory cytokines, such as IL-1 $\beta$, IL-6, and TNF- $\alpha$, have different roles in cancer, and they can be associated with fatigue, depression, cognitive impairment, cachexia, anorexia, and pain (all of which 
negatively affect the quality of life). They can influence the metabolism of certain anticancer drugs by acting on cytochrome P450 enzymes and can lead to the activation of $\mathrm{NF}-\kappa \mathrm{B}$, which enables the survival of certain cancer cells and provides a mechanism by which they might become resistant to chemotherapy and radiotherapy. ${ }^{24} \mathrm{Clinical}$ investigations, however, suggest that L-MTP-PE might result in a net positive effect in patients treated for osteosarcoma. ${ }^{3,25}$

\section{Mifamurtide and osteosarcoma}

The extremely rare phenomenon of spontaneous regression of malignancies is often associated with feverish infections. ${ }^{26}$ Results from a recent investigation on osteosarcoma suggested increased survival for patients who experienced deep postoperative infections. ${ }^{27}$ In this retrospective analysis, 41 patients who developed a deep infection within 1 year after the implantation of a graft had 10-year survival rate of $84.5 \%$, compared with $62.3 \%$ in the cohort of 338 patients who did not have a deep infection. ${ }^{27}$ Clearly, these results have to be interpreted with caution. They are, however, of interest, especially in the context of immunotherapy for osteosarcoma.

The rationale behind the use of L-MTP-PE in osteosarcoma treatment is to mimic a kind of infection that can help to eradicate residual micrometastases that are not eliminated by adjuvant chemotherapy. A landmark investigation in dogs provided first evidence that L-MTP-PE may be useful in the treatment of osteosarcoma. ${ }^{28}$ Osteosarcoma in dogs has many similarities to that in humans, and dogs with spontaneous osteosarcoma had improved disease-free survival when treated after surgery with L-MTP-PE when compared with dogs receiving placebo. ${ }^{28}$ The results from this randomized study and subsequent investigations in dogs and other animals prompted clinical studies in humans. . $^{3,29,30}$

\section{Clinical investigations with mifamurtide in patients with osteosarcoma}

A phase I clinical trial with L-MTP-PE was carried out in 37 patients with advanced cancer, and the optimum IV dose was found to be in the range of $1-4 \mathrm{mg} / \mathrm{m}^{2}$ twice weekly for 4 weeks - a dose that was well tolerated. ${ }^{31}$

A phase II clinical trial using L-MTP-PE was undertaken in patients with osteosarcoma (aged 9-59 years) and synchronous or metachronous lung metastases to determine whether L-MTP-PE therapy could improve the progressionfree interval in this high-risk group of patients. ${ }^{32}$ After a complete surgical remission was achieved, a dosage of $2 \mathrm{mg} / \mathrm{m}^{2}$ L-MTP-PE was infused over a 1-hour period twice a week for 12 weeks in 1 group of 12 patients ( 24 doses). The second group of 16 patients received $2 \mathrm{mg} / \mathrm{m}^{2} \mathrm{~L}-\mathrm{MTP}-\mathrm{PE}$ twice a week for 12 weeks, then once a week for an additional 12 weeks for a total of 24 weeks of therapy ( 36 doses). Relapse-free survival in these 2 groups was compared with a matched historical control group (21 patients with metachronous lung metastases, who received adjuvant chemotherapy); patients who received 24 weeks of L-MTP-PE therapy had a prolongation in time to relapse ( 9 months compared with 4.5 months for the historical control group), but those who received 12 weeks of therapy did not. ${ }^{32}$ Although the comparison with a historical control group does not allow definitive conclusions, these data suggested that L-MTP-PE deserved further investigation in patients with osteosarcoma in a more appropriate adjuvant setting. Of note, there was no evidence that L-MTP-PE therapy caused an acceleration of relapse, and toxic reactions were limited to chills, fever, headache, myalgias, and fatigue, which were especially common during the first administration. Subsequent administrations yielded minimal or no toxic effects, except for delayed fatigue (grade 1). ${ }^{32}$

In this phase II trial, additional biological investigations were performed. For example, in 5 patients, a single osteosarcoma metastasis recurred within 6 weeks after the completion of L-MTP-PE therapy. The nodules were removed, and the specimens were compared with lung metastasis specimens obtained before L-MTP-PE therapy. After L-MTP-PE therapy in 3 patients, peripheral fibrosis surrounded the lung metastasis, and inflammatory cell infiltration and neovascularization were present. This is in contrast to lung metastases removed following chemotherapy, which showed central necrosis with viable peripheral tumor cells and no signs of inflammatory response. In the fourth case, evidence of early fibrotic changes was found. This and the fifth case showed a change in malignant characteristics, from high grade before L-MTP-PE therapy to low grade after therapy. Based on the results in these 5 patients, it was concluded that there is evidence for a biological effect of L-MTP-PE on osteosarcoma lung metastases. ${ }^{22}$

In a subsequent phase IIb trial, the tolerability of L-MTP-PE given in combination with ifosfamide was investigated in 9 patients with osteosarcoma and lung metastases. ${ }^{33}$ It was demonstrated that the simultaneous administration of an immunostimulator (ie, L-MTP-PE) and a cytotoxic agent (ie, ifosfamide) was safe and did not cause an increase in toxicity or obliteration of the immune response. ${ }^{33}$

The results of the trials described earlier prompted a phase III randomized prospective trial, the intergroup (INT) 
study 0133 from the Children's Cancer (CCG 7921) and Pediatric Oncology (POG 9351) Groups (now merged to the Children's Oncology Group [COG]), in patients with newly diagnosed osteosarcoma aged $\leq 30$ years.

\section{Intergroup study 0133 (CCG 7921 and POG 935I)}

This largest ever completed randomized trial in osteosarcoma was conducted from 1993 to 1997 and recruited a total of 662 patients with nonmetastatic osteosarcoma whose primary tumors were considered to be resectable. ${ }^{25}$ In addition, 91 patients with primary metastatic osteosarcoma were recruited and also analyzed. ${ }^{34}$

In INT 0133, all patients were intended to receive a similar backbone treatment (high-dose methotrexate with leucovorin rescue + doxorubicin $/ a$ driamycin + cisplatin $=M A P$ ) with identical cumulative doses of high-dose methotrexate (12 times at doses of $12 \mathrm{~g} / \mathrm{m}^{2}$ ), doxorubicin (6 times at doses of $75 \mathrm{mg} / \mathrm{m}^{2}$ ), and cisplatin (4 times at doses of $120 \mathrm{mg} / \mathrm{m}^{2}$ ). The randomized prospective study was conducted with a $2 \times 2$ factorial design with 4 treatment arms: (1) arm A-consisting of MAP, (2) arm A+ consisting of MAP + L-MTP-PE, (3) arm B- consisting of MAP + ifosfamide, and (4) arm B+ consisting of MAP + ifosfamide + L-MTP-PE. Ifosfamide was given 5 times at a dose of $9 \mathrm{~g} / \mathrm{m}^{2}$ per course. L-MTP-PE was given at a dose of $2 \mathrm{mg} / \mathrm{m}^{2}$, and the dose was escalated up to $2 \mathrm{mg} / \mathrm{m}^{2}+2 \mathrm{mg}$ if no biological activity (ie, cytokine flu) was observed after the first administration. L-MTP-PE was administered IV twice weekly for 12 weeks beginning at week 12 and then weekly for additional 24 weeks beginning at week 24 . The duration of treatment was 20 weeks for patients randomly assigned to arm A-, 27 weeks for patients randomly assigned to arm $\mathrm{B}-$, and 36 weeks for patients randomly assigned to arm $\mathrm{A}+$ and $\operatorname{arm} \mathrm{B}+{ }^{25,35}$

The results of INT 0133 were analyzed after different observation durations and published in 2005 and $2008 . .^{25,35} \mathrm{In}$ the first analysis, Meyers et al ${ }^{35}$ identified event-free survival (EFS) as the primary outcome measure and reported a significant interaction between the L-MTP-PE and ifosfamide with respect to the primary end point EFS. ${ }^{36}$ Moreover, no significant impact of L-MTP-PE on EFS was identified. ${ }^{35}$ The focus on EFS in this initial report was later quoted as an error, and in the subsequent analysis with longer follow-up, OS and EFS were reported as the end points of the study. ${ }^{25}$ This reanalysis with longer follow-up reported improved survival with the addition of L-MTP-PE to chemotherapy (pooled analysis) from $70 \%$ to $78 \% 6$-year OS $(P=0.03$; relative risk, 0.73). ${ }^{25}$ In the analysis of EFS, a $P$-value of
0.102 for the test of interaction was interpreted as sufficient evidence of no interaction, and the pooled analysis of EFS was also carried out. ${ }^{25}$ However, only very large interactions could be detected, so a borderline $P$ value - as presented herein for EFS - should not be interpreted as evidence of no interaction. ${ }^{36}$ An unpooled analysis of EFS showed a strong difference between treatment without and with L-MTP-PE for arm B and almost identical outcomes with and without L-MTP-PE for arm A. Thus, the pooled analysis could have produced estimates of L-MTP-PE treatment effects that were either too large or too small, depending on whether ifosfamide was present. For OS, there was less evidence for an interaction and a borderline significant effect of L-MTP-PE $(P=0.03)$ in the pooled analysis. Overall, the effect of treatment on OS is expected to be mediated through EFS, and this, amongst other issues, makes the interpretation of the results of INT 0133 very difficult (for details, see Hunsberger et $\mathrm{al}^{36}$ and Bielack et $\mathrm{a}^{37}$ ). In the cohort of patients with primary metastatic osteosarcoma, the addition of L-MTP-PE to chemotherapy did not achieve a statistically significant improvement in the outcome. ${ }^{34}$ There was, however, a trend toward better outcomes in patients with synchronous metastases treated with L-MTP-PE, but the low number of patients analyzed precluded clear conclusions.

\section{Clinical safety}

Almost all patients (and healthy volunteers) treated with L-MTP-PE experienced chills, fever, headache, myalgias, and fatigue, especially during the first administration., ${ }^{3,10}$ These adverse drug reactions, however, decreased in intensity and were often no longer observed in subsequent administrations. Caution must be noted in patients with pre-existing asthma, as these patients can develop mild to moderate respiratory distress associated with treatment. Other concerns include hearing loss and delayed fatigue. Exhaustive data on the safety and tolerability of L-MTP-PE are available at the Web site of the EMA (http://www.ema.europa.eu/humandocs/ PDFs/EPAR/mepact/H-802-PI-en.pdf; accessed March 10, 2010).

\section{Regulatory status}

MEPACT (L-MTP-PE) has recently received marketing authorization in the EU by the EMA, whereas no approval has yet been provided by the US Food and Drug Administration in the United States. In Europe, based on the assessment of more than 80 studies, the Committee for Medicinal Products for Human Use suggested that the benefits of MEPACT are greater than its risks when used in combination with adjuvant 
chemotherapy to treat high-grade resectable nonmetastatic osteosarcoma after the complete macroscopic surgical resection. The committee recommended that MEPACT be given marketing authorization, and the European Commission granted a marketing authorization for MEPACT, valid throughout the EU, on March 6, 2009 (detailed report available at http://www.ema.europa.eu/humandocs/ PDFs/EPAR/mepact/H-802-PI-en.pdf; accessed March 10, 2010), to be used as adjuvant to polychemotherapy for the postoperative treatment of patients (aged 2-30 years) with nonmetastatic high-grade resectable osteosarcoma after the complete macroscopic resection.

\section{Expected costs of the treatment with MEPACT}

MEPACT is currently produced by Takeda and is available since 2010 for clinical use in certain countries (eg, Germany and Austria). The cost for one vial containing $4 \mathrm{mg}$ L-MTP-PE will be around $€ 3,000$. The agent is intended to be given 48 times at a dose of $2 \mathrm{mg} / \mathrm{m}^{2}$, and the estimated costs of the drug alone for the treatment of 1 patient in Germany are about $€ 110,000,00$ for children and $€ 150,000,00$ for adults (http://www.akdae. de/30/10/50/2009004-Mepact.pdf). Overall, this would probably approximately double the costs for the treatment of a patient with osteosarcoma.

\section{Summary}

Based on the available preclinical and clinical data, L-MTP-PE is an agent that deserves further investigation to define its role in the treatment of osteosarcoma. However, considerable debate about whether the addition of L-MTP-PE to standard chemotherapy regimens as an adjuvant in the treatment of patients with high-grade osteosarcoma truly results in an unequivocally proven benefit is still ongoing, and as outlined above, the results of the randomized prospective clinical trial INT 0133 did not suffice to resolve this debate. ${ }^{36,37}$ The investigators of INT-0133 state that they did not discuss or advocate any specific use of L-MTP-PE, but that they think it is important to place L-MTP-PE into some perspective. ${ }^{38}$ Lead investigators of the largest osteosarcoma study groups and the National Institute of Health agree that L-MTP-PE is an agent that warrants additional investigation before it can be considered for routine use in the frontline therapy of patients with high-grade osteosarcoma. ${ }^{37}$ Therefore, future international prospective trials should further investigate L-MTP-PE in osteosarcoma.

\section{Disclosures}

Leo Kager and Ulrike Pötschger report no conflicts of interest in this work. Stefan Bielack has acted as a consultant for IDM, Roche, and Takeda Millenium, and as an Advisory Board member for Merck.

\section{References}

1. Mirabello L, Troisi RJ, Savage SA. Osteosarcoma incidence and survival rates from 1973 to 2004: data from the Surveillance, Epidemiology, and End Results Program. Cancer. 2009;115:1531-1543.

2. Mirabello L, Troisi RJ, Savage SA. International osteosarcoma incidence patterns in children and adolescents, middle ages and elderly persons. Int J Cancer. 2009;125(1):229-234.

3. Meyers PA. Muramyl tripeptide (mifamurtide) for the treatment of osteosarcoma. Expert Rev Anticancer Ther. 2009;9:1035-1049.

4. Kager L, Zoubek A, Potschger U, et al. Primary metastatic osteosarcoma: presentation and outcome of patients treated on neoadjuvant Cooperative Osteosarcoma Study Group protocols. J Clin Oncol. 2003;21:2011-2018.

5. Kempf-Bielack B, Bielack SS, Jurgens H, et al. Osteosarcoma relapse after combined modality therapy: an analysis of unselected patients in the Cooperative Osteosarcoma Study Group (COSS). J Clin Oncol. 2005;23:559-568.

6. Bielack SS, Kempf-Bielack B, Delling G, et al. Prognostic factors in high-grade osteosarcoma of the extremities or trunk: an analysis of 1,702 patients treated on neoadjuvant cooperative osteosarcoma study group protocols. J Clin Oncol. 2002;20:776-790.

7. Bielack SS, Kempf-Bielack B, Branscheid D, et al. Second and subsequent recurrences of osteosarcoma: presentation, treatment, and outcomes of 249 consecutive cooperative osteosarcoma study group patients. J Clin Oncol. 2009;27:557-565.

8. Picci P, Sangiorgi L, Rougraff BT, et al. Relationship of chemotherapyinduced necrosis and surgical margins to local recurrence in osteosarcoma. J Clin Oncol. 1994;12:2699-2705.

9. Mori K, Ando K, Heymann D. Liposomal muramyl tripeptide phosphatidyl ethanolamine: a safe and effective agent against osteosarcoma pulmonary metastases. Expert Rev Anticancer Ther. 2008;8: $151-159$.

10. Anderson P. Liposomal muramyl tripeptide phosphatidyl ethanolamine: ifosfamide-containing chemotherapy in osteosarcoma. Future Oncol. 2006;2:333-343

11. Nardin A, Lefebvre ML, Labroquere K, et al. Liposomal muramyl tripeptide phosphatidylethanolamine: targeting and activating macrophages for adjuvant treatment of osteosarcoma. Curr Cancer Drug Targets. 2006;6:123-133.

12. Balkwill F. Tumour necrosis factor and cancer. Nat Rev Cancer. 2009;9:361-371.

13. Geddes K, Magalhaes JG, Girardin SE. Unleashing the therapeutic potential of NOD-like receptors. Nat Rev Drug Discov. 2009;8: $465-479$.

14. Shaw MH, Reimer T, Kim YG, et al. NOD-like receptors (NLRs): bona fide intracellular microbial sensors. Curr Opin Immunol. 2008;20: 377-382.

15. Marina-Garcia N, Franchi L, Kim YG, et al. Pannexin-1-mediated intracellular delivery of muramyl dipeptide induces caspase-1 activation via cryopyrin/NLRP3 independently of Nod2. J Immunol. 2008; 180:4050-4057.

16. Sone S, Mutsuura S, Ogawara M, et al. Potentiating effect of muramyl dipeptide and its lipophilic analog encapsulated in liposomes on tumor cell killing by human monocytes. J Immunol. 1984;132:2105-2110.

17. Fidler IJ, Brown NO, Hart IR. Species variability for toxicity of free and liposome-encapsulated muramyl peptides administered intravenously. J Biol Response Mod. 1985;4:298-309. 
18. Murray JL, Kleinerman ES, Cunningham JE, et al. Phase I trial of liposomal muramyl tripeptide phosphatidylethanolamine in cancer patients. J Clin Oncol. 1989;7:1915-1925.

19. Landmann R, Obrist R, Denz H, et al. Pharmacokinetics and immunomodulatory effects on monocytes during prolonged therapy with liposomal muramyltripeptide. Biotherapy. 1993;7:1-12.

20. Fidler IJ, Barnes Z, Fogler WE, et al. Involvement of macrophages in the eradication of established metastases following intravenous injection of liposomes containing macrophage activators. Cancer Res. 1982;42: 496-501.

21. Fidler IJ, Poste G. Macrophage-mediated destruction of malignant tumor cells and new strategies for the therapy of metastatic disease. Springer Semin Immunopathol. 1982;5:161-174.

22. Kleinerman ES, Jia SF, Griffin J, et al. Phase II study of liposomal muramyl tripeptide in osteosarcoma: the cytokine cascade and monocyte activation following administration. J Clin Oncol. 1992;10: 1310-1316.

23. Asano T, Kleinerman ES. Liposome-encapsulated MTP-PE: a novel biologic agent for cancer therapy. J Immunother Emphasis Tumor Immunol. 1993;14:286-292.

24. Seruga B, Zhang H, Bernstein LJ, et al. Cytokines and their relationship to the symptoms and outcome of cancer. Nat Rev Cancer. 2008;8: 887-899.

25. Meyers PA, Schwartz CL, Krailo MD, et al. Osteosarcoma: the addition of muramyl tripeptide to chemotherapy improves overall survival - a report from the Children's Oncology Group. J Clin Oncol. 2008;26:633-638.

26. Hobohm U. Fever therapy revisited. Br J Cancer. 2005;92:421-425.

27. Jeys LM, Grimer RJ, Carter SR, et al. Post operative infection and increased survival in osteosarcoma patients: are they associated? Ann Surg Oncol. 2007;14:2887-2895.

28. MacEwen EG, Kurzman ID, Rosenthal RC, et al. Therapy for osteosarcoma in dogs with intravenous injection of liposome-encapsulated muramyl tripeptide. J Natl Cancer Inst. 1989;81:935-938.
29. Kurzman ID, MacEwen EG, Rosenthal RC, et al. Adjuvant therapy for osteosarcoma in dogs: results of randomized clinical trials using combined liposome-encapsulated muramyl tripeptide and cisplatin. Clin Cancer Res. 1995;1:1595-1601.

30. Kurzman ID, Shi F, Vail DM, et al. In vitro and in vivo enhancement of canine pulmonary alveolar macrophage cytotoxic activity against canine osteosarcoma cells. Cancer Biother Radiopharm. 1999;14:121-128.

31. Creaven PJ, Cowens JW, Brenner DE, et al. Initial clinical trial of the macrophage activator muramyl tripeptide-phosphatidylethanolamine encapsulated in liposomes in patients with advanced cancer. $J$ Biol Response Mod. 1990;9:492-498.

32. Kleinerman ES, Gano JB, Johnston DA, et al. Efficacy of liposomal muramyl tripeptide (CGP 19835A) in the treatment of relapsed osteosarcoma. Am J Clin Oncol. 1995;18:93-99.

33. Kleinerman ES, Meyers PA, Raymond AK, et al. Combination therapy with ifosfamide and liposome-encapsulated muramyl tripeptide: tolerability, toxicity, and immune stimulation. J Immunother Emphasis Tumor Immunol. 1995;17:181-193.

34. Chou AJ, Kleinerman ES, Krailo MD, et al. Addition of muramyl tripeptide to chemotherapy for patients with newly diagnosed metastatic osteosarcoma: a report from the Children's Oncology Group. Cancer. 2009; 115:5339-5348

35. Meyers PA, Schwartz CL, Krailo M, et al. Osteosarcoma: a randomized, prospective trial of the addition of ifosfamide and/or muramyl tripeptide to cisplatin, doxorubicin, and high-dose methotrexate. J Clin Oncol. 2005;23:2004-2011.

36. Hunsberger S, Freidlin B, Smith MA. Complexities in interpretation of osteosarcoma clinical trial results. J Clin Oncol. 2008;26:3103-3104; author reply 3104-3105.

37. Bielack SS, Marina N, Ferrari S, et al. Osteosarcoma: the same old drugs or more? J Clin Oncol. 2008;26:3102-3103; author reply 3104-3105.

38. Meyers PA, Schwartz CL, Krailo M, et al. In reply. J Clin Oncol. 2008;26(18):3104-3105.
Therapeutics and Clinical Risk Management

\section{Publish your work in this journal}

Therapeutics and Clinical Risk Management is an international, peerreviewed journal of clinical therapeutics and risk management, focusing on concise rapid reporting of clinical studies in all therapeutic areas outcomes, safety, and programs for the effective, safe, and sustained use of medicines. This journal is indexed on PubMed Central, CAS,

\section{Dovepress}

EMBase, Scopus and the Elsevier Bibliographic databases. The manuscript management system is completely online and includes a very quick and fair peer-review system, which is all easy to use. Visit http://www.dovepress.com/testimonials.php to read real quotes from published authors. 